\title{
MAXIMAL INTERSECTING FAMILIES OF FINITE SETS AND $n$-UNIFORM HJELMSLEV PLANES
}

\author{
DAVID A. DRAKE ${ }^{1}$ AND SHARAD S. SANE
}

\begin{abstract}
The following theorem is proved. The collection of lines of an $n$-uniform projective Hjelmslev plane is maximal when considered as a collectiion of mutually intersecting sets of equal cardinality.
\end{abstract}

1. Introduction. A clique of $k$-sets is a collection of mutually intersecting sets of size $k$. We write $N(k)$ to denote the minimum cardinality of a maximal clique of $k$-sets. Apparently the exact value of $N(k)$ is known only for very small values of $k$. However, Erdös and Lovàsz [7] have obtained the asymptotic lower bound $N(k) \geqslant$ $(8 k / 3)-3$; and Füredi [8, p. 283] writes that he can prove $N(k)<k^{f(k)}$ where $f(k)=c k^{7 / 12}$.

For particular values of $k$, the preceding upper bound can be greatly sharpened. It is easily proved, for example, that

(1.1) a projective plane of order $r$ is a maximal clique. Consequently

(1.2) $N(r+1) \leqslant r^{2}+r+1$ whenever $r$ is the order of a projective plane.

In addition Füredi has proved the following two theorems (Proposition 1 and Theorem 1 in [8]). (Füredi informs us that (1.3) is joint work with L. Babai.)

(1.3) $N\left(r^{2}+r\right) \leqslant r^{4}+r^{3}+r^{2}$ whenever $r$ is the order of a projective plane.

(1.4) $N(2 r) \leqslant 3 r^{2}$ whenever $r$ is the order of a projective plane.

In this paper we obtain the following common generalization of (1.2) and (1.3).

THEOREM 1.1. If $r$ is the order of a finite projective plane, then $N\left(r^{n}+r^{n-1}\right) \leqslant r^{2 n}$ $+r^{2 n-1}+r^{2 n-2}$ for every positive integer $n$.

Füredi proves (1.3) by constructing a 2-uniform projective Hjelmslev plane over an arbitrary finite projective plane and then observing that such Hjelmslev planes are maximal cliques. Henceforth we write $\mathrm{PH}$-plane for projective Hjelmslev plane. The more difficult of the two steps in the Füredi program is the PH-plane construction, a construction which has been discovered independently by Füredi [8] and Craig [3] (see also Lüneburg [13]). Since the class of 1-uniform PH-planes is by definition just the class of finite projective planes, conclusions (1.2) and (1.3) both follow by observing that the line set of every $n$-uniform PH-plane with $n=1$ or 2 is a maximal clique. Similarly we shall obtain Theorem 1.1 as a corollary to the following result.

Received by the editors January 18, 1982.

1980 Mathematics Subject Classification. Primary 05B30; Secondary 05B25, 51E30.

${ }^{1}$ Support by the National Science Foundation (grant no. MCS-7903166) is gratefully acknowledged. 
THEOREM 1.2. The line set of every ( finite) $n$-uniform projective Hjelmslev plane is a maximal clique.

The contribution of this paper is to prove Theorem 1.2. The other step, that of establishing the existence of $n$-uniform PH-planes over arbitrary projective planes, has already been completed: first by Artmann [1] and later by Drake [6] who used a different construction.

If $k$ can be represented both as $r^{m}+r^{m-1}$ and as $s^{p}+s^{p-1}$ with $m<p$, one should apply Theorem 1.1 with $n=p$ to obtain the sharper bound. Unfortunately such double representations occur for prime powers $r$ and $s$ only when $m=1$ and in the case $2^{3}+2^{2}=3^{2}+3=11+1$. In the latter case one obtains $N(12) \leqslant 133$ by using (1.2), $N(12) \leqslant 117$ by using the Füredi result $(1.3)$, and $N(12) \leqslant 112$ by using Theorem 1.1 with $n=3$. The real value of Theorem 1.1, of course, is that variation in $n$ allows one to obtain a bound for $N(k)$ for new values of $k$.

2. Prerequisites. We refer the reader to [5, pp. 192-197] for background material that includes the definitions of PH-planes and NAH-planes (near affine Hjelmslev planes). We repeat here some of the material from the cited pages, however, because the conclusions of this paper will interest a number of mathematicians without previous knowledge of Hjelmslev planes. We use the designation $H$-planes to refer collectively to NAH- and PH-planes.

To every H-plane $E$ is associated a canonical (incidence-structure) epimorphism $\phi: E \rightarrow E^{\prime}$ where $E^{\prime}$ is a projective plane if $E$ is a PH-plane and an affine plane if $E$ is an NAH-plane. Points $P$ and $Q$ (lines $g$ and $h$ ) are called neighbors, and one writes $P \sim Q(g \sim h)$, if and only if $P^{\phi}=Q^{\phi}\left(g^{\phi}=h^{\phi}\right)$. One writes $\nsim$ for the negation of $\sim$. Intersecting lines $g$ and $h$ satisfy $g \sim h$ if and only if $|g \cap h|>1$. We write $(P)$ to denote the set $\{Q: Q \sim P\}$ and $(g)$ to denote the set $\{h: h \sim g\}$. The following result was proved by Klingenberg [10, Satz 3.6]. (See also the remarks on page 260 of [12].)

Proposition 2.1. Let the incidence structure $A=A(H, h)$ be obtained from $a$ PH-plane $H$ by removing a neighbor class $(h)$ of lines as well as all points of $H$ which are incident with lines of $(h)$. Then $A$ is an NAH-plane.

To each finite H-plane $E$ are associated three integers denoted by $r, s$ and $t$. For any flag $(P, g)$ the integer $t$ is the number of lines $h$ through $P$ which satisfy $h \sim g$ (as well as the number of points $Q$ on $g$ that satisfy $Q \sim P) ;|(P)|=|(g)|=t^{2}$; $s+t$ is the number of lines incident with $P$; and $r$ is the order of $E^{\prime}$. Every line contains $s+t$ points if $E$ is a PH-plane, $s$ points if $E$ is an NAH-plane. The equality $s=r t$ holds for all H-planes. The preceding properties of $r, s$ and $t$ were first noted (for PH-planes only) by Kleinfeld [9]. Accordingly we shall designate this collection of properties the Kleinfeld Counting Lemma.

A nearly 1-uniform $\mathrm{PH}$-plane (NAH-plane) is a finite projective plane (finite affine plane). For $n>1$ a finite H-plane $E$ (of either type) is called nearly n-uniform if, for every point $P$, (1) $E$ induces an incidence structure $A(P)$ on $(P)$ which is a nearly ( $n-1)$-uniform NAH-plane, (2) every line of $A(P)$ is induced by $d$ lines of $E$ for 
some fixed integer $d$. Proposition 1.10(11) of [5] asserts that $d=r$. A nearly $n$-uniform $\mathrm{H}$-plane is said to be $n$-uniform if every $A(P)$ is an $(n-1)$-uniform NAH-plane with a "parallelism," but the reader will not need to understand this notion.

We now establish some conventions. All H-planes in this paper are assumed to be nearly $n$-uniform for some $n$. The symbols $E_{n}, H_{n}$ and $A_{n}$ denote a nearly $n$-uniform H-, PH- and NAH-plane, respectively, with $E^{\prime}, H^{\prime}$ and $A^{\prime}$ as the respective underlying planes. In all cases the order of the underlying plane is assumed to be $r$.

One writes $P(\simeq i) Q$ to mean that $P$ and $Q$ are joined by precisely $r^{i}$ lines for $0 \leqslant i<n$ and $P(\simeq n) Q$ to mean that $P=Q$. One writes $P(\sim i) Q$ if $P(\simeq j) Q$ for some $j \geqslant i$. The negation of $P(\sim i) Q$ is denoted by $P(\nsim i) Q$. The following result is part of Proposition 1.10 of [5]; most of the proof, however, is given in the proof of Proposition 2.2 in [4] rather than in [5].

PROPOSITION 2.2. Every nearly n-uniform $\mathrm{H}$-plane $E_{n}$ has the following properties.

(1) $s=r^{n}, t=r^{n-1}$.

(2) If $P$ and $Q$ are distinct points of $E_{n}$, then $P(\simeq i) Q$ for some nonnegative integer $i<n$.

(3) The dual of (2) holds for intersecting lines.

(4) If $P$ is in $g$ and $i \geqslant 1$, then $|\{Q \in g: Q(\sim i) P\}|=r^{n-i}$.

(5) The dual of (4) holds.

One of the principal results of [4] (Proposition 4.6) asserts that the dual of a "strongly" $n$-uniform PH-plane is a strongly $n$-uniform PH-plane. In [14, Satz 1] Törner proves that every nearly $n$-uniform PH-plane is a strongly $n$-uniform PHplane; Theorem 2.3 below is an immediate consequence. (An alternative proof is given in [11].)

THEOREM 2.3. Every nearly n-uniform PH-plane is n-uniform, and the dual of an n-uniform $\mathrm{PH}$-plane is an n-uniform $\mathrm{PH}$-plane.

Two lines $g$ and $h$ of $A_{n}$ are said to be quasiparallel (and one writes $g \mid h$ ) if $g^{\phi} \| h^{\phi}$ in $A^{\prime}$. Then | is an equivalence relation which partitions the lines of $A_{n}$ into $r+1$ quasiparallel classes; each such class is the disjoint union of $r$ neighbor classes of lines, hence consists of $r t^{2}$ lines. As observed in [5, p. 202], the condition $g \mid h$ holds if and only if $|g \cap h| \neq 1$. This characterization of the quasiparallel relation makes it easy to prove the following lemma.

LEMMA 2.4. Let $g, h$ and $P$ be lines and point of $E_{n}$ such that $g^{\prime}=g \cap(P)$ and $h^{\prime}=h \cap(P)$ are not empty. Then $g \sim h$ if and only if $g^{\prime} \mid h^{\prime}$ in $A(P)$.

\section{Preliminary results.}

Proposition 3.1. Let $\Lambda$ be a quasiparallel class of $A_{n}, S \subset \Lambda,|S|<s=r^{n}$. Then there is a set $C$ of points of $A_{n}$ which has the following properties: (1) $|C|=s ;(2)$ each pair of points of $C$ is joined by a line of $\Lambda$; (3) no point of $C$ lies on any line of $S$. 
Proof. For $n=1, \Lambda$ is a parallel class, and $C$ may be taken to be the set of points of any line in $\Lambda \backslash S$. Assume $n>1$, and let $\Lambda_{1}, \Lambda_{2}, \ldots, \Lambda_{r}$ be the $r$ line neighborhoods contained in $\Lambda$. If $S_{j}$ denotes $S \cap \Lambda_{j}$ for each $j$, then $\left|S_{i}\right|<s / r=t$ for some $i$. We intend to obtain $C$ from the set of points that are incident with lines of $\Lambda_{i}$. Let $h$ be a line in $\Lambda_{i} ; P_{1}, P_{2}, \ldots, P_{r}$ be $r$ mutually nonneighbor points on $h$. For arbitrary fixed $j$, let $\Lambda^{\prime}=\left\{g^{\prime}: g^{\prime}=g \cap\left(P_{j}\right)\right.$ for some $g$ in $\left.\Lambda_{i}\right\}, S^{\prime}=\left\{g^{\prime}: g^{\prime}=g \cap\left(P_{j}\right)\right.$ for some $g$ in $S_{i}$. By Lemma $2.4, \Lambda^{\prime}$ is a quasiparallel class of lines in the nearly $(n-1)$-uniform NAH-plane $A\left(P_{j}\right)$ : and $S^{\prime}$ is a subset of fewer than $t=r^{n-1}$ lines of $\Lambda^{\prime}$. By the induction assumption there is a set $C_{j} \subset\left(P_{j}\right)$ such that $(1)\left|C_{j}\right|=r^{n-1}$; (2) each pair of points of $C_{j}$ is joined by a line of $\Lambda$; (3) no point of $C_{j}$ lies on any line of $S$. We take $C$ to be the union of the $C_{j}$.

Proposition 3.2. Let $g$ be any line of $H_{n}, N \subset(g),|N|<t$. Then there is a set $D$ of points of $H_{n}$ with the properties: (1) $|D|=s+t$; (2) each pair of points of $D$ is joined by a line of $(g) ;(3)$ no point of $D$ lies on any line of $N$.

Proof. Let $P_{0}, P_{1}, \ldots, P_{r}$ be $r+1$ mutually nonneighbor points on $g$. For fixed $j \geqslant 0$, apply Lemma 2.4 to see that the lines of $N$ induce a subset $N^{\prime}$ of a quasiparallel class of lines in $A\left(P_{j}\right)$. Applying Proposition 3.1 (with $n-1$ instead of $n$ ), we obtain a set $D_{j}$ of points of $\left(P_{j}\right)$ such that $(1)\left|D_{j}\right|=t ;(2)$ each pair of points of $D_{j}$ is joined by a line of $(g)$; (3) no point of $D_{j}$ lies on any line $N$. We now take $D$ to be the union of the $D_{j}$.

Proposition 3.3. Let $S$ be a set of at most $s+t$ mutually intersecting lines of $A_{n}$ whose union contains every point of $A_{n}$. Then all lines of $S$ pass through a common point.

Proof. The assertion is easily verified for $n=1$, so assume $n>1$. Let $g_{1}^{\prime}, g_{2}^{\prime}, \ldots, g_{d}^{\prime}$ be the distinct images in $A^{\prime}$ of the lines of $S$. Since the $g_{i}^{\prime}$ intersect in $A^{\prime}, d \leqslant r+1$. Then the $g_{i}^{\prime}$ pass through a common point $P^{\prime}$, and hence the lines of $S$ all contain points from a common neighborhood $(P)$. The number of points of $A_{n}$ not in $(P)$ is $t^{2}\left(r^{2}-1\right)=s^{2}-t^{2}$, and each line of $S$ contains $s-t$ points outside $(P)$. Then every point outside $(P)$ must lie on a single line of $S$, so every pair of lines of $S$ must intersect in $(P)$. Let $g$ be any line of $S$. Applying Proposition 2.2(5) with $i=n-1$, one sees that there are $r-1$ other lines $h$ which satisfy $h \cap(P)=g \cap(P)$. Take $Q$ to be any point of $h \backslash(P)$, and let $k$ be a line of $S$ which contains $Q$. Then $k$ and $g$ intersect in $g \cap(P)=h \cap(P)$. Then $k \cap h$ contains nonneighbor points, so $h=k$ is in $S$. It follows that the set $S^{*}=\{g \cap(P): g \in S\}$ has cardinality at most $(s+t) / r=r^{n-1}+r^{n-2}$. Applying the induction assumption to $A(P)$, we see that all lines of $S^{*}$ (and therefore all lines of $S$ ) meet in a common point.

4. Proofs of the main results. Thanks to Theorem 2.3 , it is immaterial whether we prove Theorem 1.2 or its dual. Then let $S$ be a set of $s+t$ or fewer lines of $H_{n}$ whose union contains every point of $H_{n}$. To complete the proof of Theorem 1.2 it suffices to prove the existence of a point $P$ which lies on all lines of $S$. We intend to apply Proposition 3.3. To do so, we must remove a neighbor class $(h)$ of lines from $H_{n}$ to 
obtain a nearly $n$-uniform NAH-plane $A_{n}$ (see Proposition 2.1). This must be done so that the intersections of lines of $S$ lie in $A_{n}$.

For any $g$ in $S$ let $N$ denote $S \cap(g)$. Assume $|N|<t$, and apply Proposition 3.2 to obtain a set $D$ of $s+t$ points. Conditions (2) and (3) of Proposition 3.2 guarantee that the points of $D$ lie on at least $s+t$ lines of $S \backslash(g)$. Since $g$ is in $S$, we have produced the contradiction $|S|>s+t$. Then $|S \cap(g)|$ must be at least $t$ for every $g$ in $S$, so $S$ contains lines from at most $(s+t) / t=r+1$ distinct line neighborhoods of $H_{n}$. Consider the image $S^{\phi}$ of $S$ in $H^{\prime}$, and apply the dual of (1.1): one sees that $S^{\phi}$ is the set of all $r+1$ lines incident with some point $Q^{\prime}$ of $H^{\prime}$. Then $S$ contains exactly $t(r+1)=s+t$ lines. Let $Q$ be a point of $H_{n}$ with $Q^{\phi}=Q^{\prime}$. The number of flags $(R, g)$ with $g$ in $S$ and $R \nsim Q$ is $(s+t) s=t^{2}\left(r^{2}+r\right)$; i.e., is just the number of points $R$ of $H_{n}$ with $R \nsim Q$. Then every point $R \nsim Q$ lies on a unique line of $S$, so all intersections of pairs of lines of $S$ lie in $(Q)$. Let $h$ be any line having an empty intersection with $(Q)$. Applying Proposition 3.3 to $A_{n}=A\left(H_{n}, h\right)$ completes the proof of Theorem 1.2.

To prove Theorem 1.1, let $r$ be the order of a projective plane, $n$ be a positive integer. Then there exists an $n$-uniform PH-plane $H_{n}$ whose associated plane $H^{\prime}$ is of order $r$ : this assertion is Corollary 8 of [6]; it is also the main theorem of [1] if one uses the Bacon result [2] that finite PH-planes of level $n$ are $n$-uniform. Now Theorem 1.1 follows from Theorem 1.2 in view of Proposition 2.2(1) and the Kleinfeld Counting Lemma.

Acknowledgment. During the writing of this paper, the second author held a visiting position in the Department of Mathematics at the University of Florida.

\section{REFERENCES}

1. B. Artmann, Existenz und projektive Limiten von Hjelmslev-Ebenen n-ter Stufe, Atti del Convegno di Geometria Combinatoria e sue Applicazioni, Perugia, 1971, pp. 27-41.

2. P. Y. Bacon, Strongly n-uniform and level $n$ Hjelmslev planes, Math. Z. 127 (1972), 1-9.

3. R. T. Craig, Extensions of finite projective planes. I. Uniform Hjelmslev planes, Canad. J. Math. 16 (1964), 261-266.

4. D. A. Drake, On n-uniform Hjelmslev planes, J. Combin. Theory 9 (1970), 267-288.

5. Existence of parallelisms and projective extensions for strongly n-uniform near affine Hjelmslev planes, Geom. Dedicata 3 (1974), 191-214.

6. C__ Constructions of Hjelmslev planes, J. Geometry 10 (1977), 179-193.

7. P. Erdös and L. Lovàsz, Problems and results on 3-chromatic hypergraphs and some related questions, Proc. Colloq. Math. Soc. J. Bolyai, no. 10, North-Holland, Amsterdam, 1974, pp. 609-627.

8. Z. Füredi, On maximal intersecting families of finite sets, J. Combin. Theory Ser. A 28 (1980), 282-289.

9. E. Kleinfeld, Finite Hjelmslev planes, Illinois J. Math. 3 (1959), 403-407.

10. W. Klingenberg, Projektive und affine Ebenen mit Nachbarelementen, Math. Z. 60 (1954), 384-406.

11. B. V. Limaye and S. S. Sane, On partial designs and m-uniform projective Hjelmslev planes, J. Combin. Inform. System Sci. 3 (1978), 223-237.

12. H. Lüneburg, Affine Hjelmslev-Ebenen mit transitiver Translationsgruppe, Math. Z. 79 (1962), 260-288.

13. __ Kombinatorik, Birkhäuser Verlag, Basel, 1971.

14. G. Törner, n-uniforme projektive Hjelmslev-Ebenen sind stark n-uniform, Geom. Dedicata 6 (1977), $291-295$.

Department of Mathematics, University of FloRida, Gainesville, Florida 32611

Department of Mathematics, University of Bombay, Santacruz (EAst), Bombay 29AS, India 